\title{
Strategic models of intermediation networks
}

\author{
Daniele Condorelli * Andrea Galeotti ${ }^{\dagger}$
}

March 24, 2015

\begin{abstract}
This chapter surveys a set of papers that analyze strategic intermediation in networks. In all these papers, the architecture of the network, by determining the level of competition and outside options of traders, has an impact on how surplus is shared across trading parties. We emphasize the insights that are most recurrent in the literature.
\end{abstract}

\section{Introduction}

A time-honored tradition in economics sees markets as populated by agents interacting anonymously through the price system. The Walrasian paradigm, which culminated in the Arrow-Debreu-McKenzie model, has contributed to our understanding of markets especially large ones. However, a body of evidence accumulated over time shows that individual relationships and bonds of trust, arising from kinship ties, geographical proximity, joint investments, and so forth, affect economic outcomes in many relevant cases. ${ }^{1}$

A number of authors have taken up the challenge of studying the implications of decentralized trade for markets. ${ }^{2}$ Kirman (1997) and Tesfatsion (1997) stand as pioneering

${ }^{*}$ Department of Economic, University of Essex. Email: dcond@essex.ac.uk

${ }^{\dagger}$ Department of Economic, University of Essex. Email: agaleo@essex.ac.uk Andrea Galeotti is grateful to the European Research Council for support through ERC-starting grant (award No. 283454) and to the Leverhulme Trust for support through the Philip Leverhulme Prize.

${ }^{1}$ For instance, the literature on social networks in sociology documents that personal relationships spill over business relationships, and vice versa; also, the importance of ethnicity ties in shaping trade relations cannot be overemphasized, even in times of globalization, e.g., see Rauch (1999).

${ }^{2}$ There is a large body of work that studies decentralized trade, in which the interaction across buyers, sellers and intermediaries is modeled through search and random matching; see the seminal work of Rubinstein and Wolinsky (1987) and Duffie et al. (2005) for a more recent work. This survey focuses on models in which the interaction among traders is represented by a graph. 
works in economics, for their modeling of markets as non-anonymous and the economy as a network. Corominas-Bosch (2003) and Kranton and Minehart (2000) developed the first strategic models of trading in networks. The key feature of these papers is that buyers and sellers have preferential relationships-i.e., links-, that make trade among them feasible. The network structure becomes relevant because it determines the outside options of a buyer and a seller bargaining over the trade surplus. The literature on buyer-seller networks has since grown to be relatively large, and it is surveyed in Chapter XX of the Handbook by Manea.

While buyer-seller networks are ubiquitous, trade in a wide range of markets involves a plethora of other subjects, such as intermediaries, dealers, brokers, market-makers, wholesalers, retailers; see, e.g., Spulber (1999). This potentially long list of middlemen, that connect producers to buyers, all contribute to the value of the final product, or at least to its delivery. Long intermediation chains play a vital role in the market for agricultural goods in developing countries, e.g., see Fafchamps and Minten (1999), as well as in financial markets for the trade of assets sold over-the-counter, e.g., see Li and Schurhoff (2012). Complex processes of production and distribution lead to supply chains, a natural example of chains of intermediaries, e.g., see Hummels et al. (2001) and Antras and Chor (2013).

The presence of intermediate agents raises a whole new set of questions: how do different network structures affect the efficiency of trading outcomes? How does the position of a trader in the network affects his payoff? How does the interplay of horizontal competition among intermediaries for buyers and sellers and the vertical complementarities in completing a chain shape the final outcome of trade? The literature we survey in this chapter addresses these questions within a common framework, in which the network architecture of intermediation plays a central role in the determination of trading outcomes.

The chapter is organized as follows. Section 2 focuses on pure intermediation networks: buyers and sellers are not directly connected and need the services of intermediaries to conclude an exchange. Intermediaries accomplish the task by buying from sellers or from other intermediaries and reselling to buyers or to other intermediaries. Intermediaries do not just match buyers and sellers or mediate the transaction; they are dealers. Intermediation networks are the norm in markets in which no centralized exchange exists such as financial over-the-counter markets and, the market for artworks and antiques. What distinguishes the papers in this section is the trading protocol through which interaction takes place: bilateral bargaining as in Condorelli and Galeotti (2012) and Manea (2013), bid-and-ask prices posted by intermediaries as in Blume et al. (2007) and Gale and Kariv (2009), and auctions as in Kotowski and Leister (2013). On a more subtle level, these papers also differ in their information structure- complete versus incomplete information. 
In the above models, it does not matter to buyers and sellers who intermediates the object, insofar as this does not alter the terms of trade. In Section 3, we relax this restriction and focus on network structure of supply chains more generally. In this case, the value of the object to the final buyers depends on who provides intermediate inputs, and the network describes how inputs from upstream firms can be combined with inputs of downstream firms. ${ }^{3}$

Recent work in economics has focused on the optimal allocation of ownership rights along a supply chain, and on stable contracts along supply chains, e.g., Antras and Chor (2013), Ostrovsky (2008) and Hatfield et al. (2013). Some other work has focused, instead, on the role that production supply chains play in translating idiosyncratic shocks into volatility at the aggregate level, e.g., Acemoglu et al. (2012). ${ }^{4}$ Here, we survey a complementary body of work that studies oligopolistic pricing in competing supply chains. The discussion is based on the work of Choi et al. (2013) and Galeotti and Goyal (2014).

The models we discuss in Section 2 and Section 3 differ in many aspects, but they generate recurrent equilibrium effects. First, the presence of intermediaries generates new form of inefficiencies when combined with information asymmetries or transaction costs. In particular, trade can occur via a long chain of intermediaries, even if shorter chains are feasible, and so intermediation costs can be too large. However, efficiency is re-established when intermediation stands as the only friction: reselling is sufficient to obtain allocative efficiency in equilibrium.

Second, market power is associated with network positions that give control and access to valuable parts of the networks. Betweenness centrality and variants of it are then adequate network proxies for market power in intermediated markets. These measures differ from classical network measures that have been used to describe power in networks, such as Bonacich and Eigenvector centrality. ${ }^{5}$ A corollary of this insight is that intermediaries can increase their market power by merging horizontally, and this comes at the expense of other traders, possibly also located further away from the merging intermediaries.

Finally, in models with complete information, the price at which the object is exchanged is increasing along the supply chain: this is necessary for intermediaries not to make a loss from buying and reselling. In contrast, when each trader's demand for the object is private information, trading conveys information, and the fact that the object remains in the market

\footnotetext{
${ }^{3}$ This also relates to recent work in macroeconomics and international trade on the role of production networks and firm-to-firm trade. The empirics of firm-to-firm trade and some modeling of production networks in macroeconomics are discussed in Chapter XX of the Handbook by Chaney.

${ }^{4}$ The effect of networks in translating idiosyncratic shock into volatility at the aggregate level is related to the topic of systemic risk, which is covered in Chapter XX of the Handbook by Acemoglu et al.

${ }^{5}$ The literature on network games shows a relation between equilibrium play and Bonacich and Eigenvector centrality. Network games are discussed in Chapter XX of the Handbook by Bramoulle and Kranton.
} 
is bad news about its value. This implies that the price at which the object is exchanged decreases over time.

\section{Intermediation}

A finite set of traders is located in a directed network and the architecture of the network is common knowledge. A link from trader $i$ to trader $j$ represents the opportunity for $i$ to sell the object to $j$. Traders can be buyers, sellers or intermediaries. Each seller owns a single indivisible unit of a durable commodity. Buyers have consumption value for the good, whereas sellers and intermediaries wish to maximize their monetary payoff.

The various models that we consider in this section are developed in the context of this general environment, but they differ in terms of the information structure and the trading protocol used to exchange the objects. We first consider a model of bilateral bargaining with random selection of proposer developed by Manea (2013). Second, we discuss the bilateral bargaining model of Condorelli and Galeotti (2012), in which each trader has a private value for the object and the seller makes all the offers. We then turn to Kotowski and Leister (2014), in which a seller uses auctions to bargain with multiple buyers or multiple intermediaries at once and to exploit competition among them. We conclude with a discussion of Blume et al. (2007) and Gale and Kariv (2009), in which intermediaries compete by posting bid-and-ask prices. The above papers occupy a central position in our survey, and we discuss related papers as we go along.

To discipline the discussion and to compare the results across models, we focus, when possible, on a specific, yet rich, class of networks.

Definition 1. A complete multipartite network is defined by a single seller s, single buyer $b$ and $a$ set $N=\{1, \ldots, n\}$ of intermediaries, connected as follows:

a. the set of intermediaries is partitioned in l-subsets, $\left\{N_{1}, \ldots, N_{L}\right\}, L \geq 1$;

b. the seller can only trade with all intermediaries in $N_{1}$; each intermediary in $N_{x}$ can only trade with all intermediaries in $N_{x+1}$, for all $x=1 . . L-1$; and each intermediary in $N_{L}$ can only trade with the final customer $b$.

Figure 1 illustrates complete multipartite networks. We refer to intermediaries in $N_{x}$ as intermediaries in tier $x$. By convention, we say that the seller belongs to tier 0 . The number of intermediaries in tier $x$ is denoted by $n_{x}$. From the view point of an intermediary in tier $x$, intermediaries in tiers $\{x+1, \ldots, L\}$ are downstream intermediaries, and those in tier 


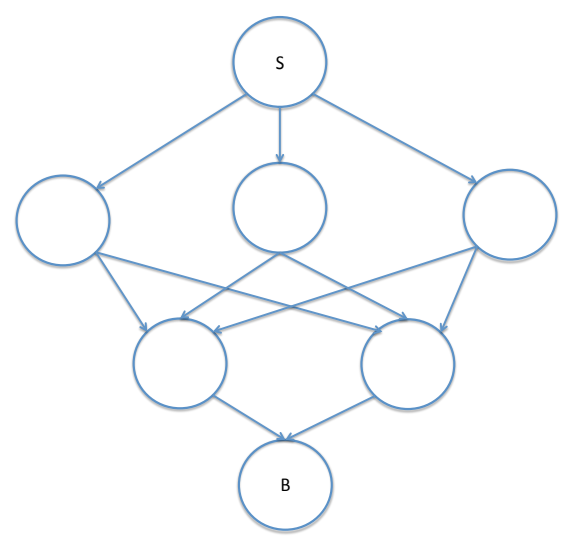

(a) Competitive network

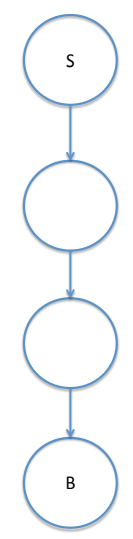

(b) Line network

Figure 1: Examples of complete multipartite networks.

$\{1, \ldots, x-1\}$ are upstream intermediaries. We say that intermediary $i$ in tier $x$ is critical if he is the sole intermediary in that tier. For each tier $x$, we denote by $k_{x}$ the number of critical downstream intermediaries. The total number of critical intermediaries in a network is denoted by $k$. The line network is a complete multipartite graph where there is only one intermediary in each tier (see Figure 1(b)). In a line network, each intermediary is critical. A competitive network is a complete multipartite network in which there are no critical intermediaries (see Figure 1(a)).

One exercise that this class of networks allows us to perform is to simulate the effect of horizontal mergers. ${ }^{6}$ For a specific trading protocol, we compute the equilibrium outcome in a network in which there are $L$ tiers with respective sizes $\left\{n_{1}, \ldots, n_{L}\right\}$. We then allow a subset of intermediaries in a tier to merge: they choose their action in order to maximize their joint profits, while all other intermediaries still act to maximize their own individual payoff. We then compare the equilibrium outcomes in these two scenarios, focusing, in particular, on the changes in realized surplus and payoffs.

\section{$2.1 \quad$ Bilateral bargaining.}

Manea (2013) develops a bilateral bargaining model with complete information, in which a single object is exchanged until it reaches a buyer along one of the possible paths of intermediaries. We now introduce a simplified version of Manea (2013) and discuss equilibrium

\footnotetext{
${ }^{6}$ Some of the papers we shall discuss also provide insights into vertical mergers. The formal discussion of these results requires introducing more notation and is beyond the scope of this survey.
} 
properties in the class of complete multipartite networks. ${ }^{7}$ We assume that there is a single buyer with consumption value $v$ for the good. The bargaining game in the network develops in an infinite number of rounds. Agents discount the future and have a common discount factor $\delta \in(0,1)$. At the beginning of round $t \geq 0$, let agent $i$ in tier $x \in\{0 \ldots L\}$ be the owner of the object ( $s$ is the owner at 0 ). Trade in period $t$ develops as follows:

1. The owner $i$ selects a trading partner, say $j$, among the intermediaries in tier $x+1$.

2. With probability $p$, the owner $i$ makes an offer to $j$, who decides whether to reject or to accept; with the complementary probability, it is agent $j$ who makes an offer to $i$.

3. If the offer is rejected, we enter period $t+1$. If the offer is accepted, trade takes place and $j$ becomes the new owner, in which case, if $j$ is an intermediary, we enter $t+1$; if $j$ is a buyer, he consumes the good and the game ends.

All actions are observed and, therefore, the game has perfect information. The focus is on stationary Markov Perfect Equilibrium (MPE): a subgame perfect equilibrium in which, at any history, all actions taken in period $t$ do not depend on actions taken in previous rounds. The main results of Manea (2013) characterize equilibrium payoffs in the limit case, in which agents become perfectly patient-i.e., $\delta \rightarrow 1$ - in general acyclic networks. For an equilibrium and a subgame in which $i$ owns the object, we define the resale value of $i$ as his expected payoff in that subgame. Manea (2013) shows that for any family of stationary MPEs, resale values converge as $\delta \rightarrow 1$ and provide an elegant recursive characterization of the limiting vector of resale values.

This characterization is based on a decomposition of the network in a sequence of layers. This sequence is constructed recursively as follows: in layer 0, we first add buyers; then we add all intermediaries linked to at least two buyers; then we add all intermediaries linked to at least two agents already included in layer 0 , and so on, until no more intermediaries are added to layer 0 . To construct layer 1 , we consider only agents that have not been assigned to layer 0 . In layer 1 , we first add intermediaries who have only one link to intermediaries in layer 0; then we add all intermediaries that have at least two links with intermediaries in layer 1; and we proceed untill we have no more intermediaries to add in layer 1 . The algorithm continues until all agents have been allocated to one layer. For example, in Figure 2.1 , the buyer is the only agent in layer 0 ; intermediary 3 is in layer 2; and all remaining agents are in layer 1 . The obtained layer structure is, in turn, what determines limiting resale values. The limiting resale value of an intermediary in layer $l$ is, in fact, $p^{l} v$.

\footnotetext{
${ }^{7}$ Manea (2013) allows for transaction costs, multiple buyers and heterogenous valuations across buyers. As we discussed below, Manea's (2013) analysis is for arbitrary acyclic networks.
} 
In the case of complete multipartite networks, this characterization takes a very simple form. Recall that $k_{x}$ is the number of critical intermediaries in tiers $\{x+1, \ldots, L\}$, while $k$ is the total number of critical intermediaries.

Proposition 1. Consider a complete multipartite network. As $\delta \rightarrow 1$ :

1. the resale value of intermediary $i$ in tier $x$ converges to $p^{k_{x}+1} v$;

2. the equilibrium payoff of the initial seller converges to $p^{k+1} v$, and the equilibrium expected payoff of the buyer converges to $(1-p) v$; and

3. the equilibrium payoff of non-critical intermediaries converges to 0 , and the equilibrium payoff of critical intermediary in tier $x$ converges to $(1-p) p^{k_{x}+1} v$.

Proposition 1 follows from the main equilibrium characterization in Manea (2013). We sketch a proof for Part 1 of Proposition 1 that uses a backward induction argument. ${ }^{8}$ When the object reaches an intermediary in the last tier, we have a standard bargaining game with random proposer and, in the limit equilibrium, the intermediary obtains payoff $p v$ and the buyer obtains $(1-p) v$. The resale value of each intermediary in tier $L$ is, then, $p v$. Suppose, now, that the object has reached intermediary $i$ in tier $L-1$. If tier $L$ is monopolized by critical intermediary $j$, then we have, again, a standard bargaining game between intermediary $i$ and intermediary $j$, where the total surplus of trade is $j$ 's resale value $p v$. In this game, intermediary $i$ obtains an expected payoff of $p^{2} v$, which is his resale value. When tier $L$ has more than one intermediary, we are in a bargaining model in which the owner, intermediary $i$, has multiple potential buyers- all the intermediaries in tier $L$, each with a resale of $p v$. Competition across intermediaries in tier $L$ implies that intermediary $i$ extracts all surplus, and so his resale value is $p v$. Part 1 of the proposition now follows, repeating this argument backwards. It is then straightforward to verify Part 2 and Part 3.

To illustrate the economic contents of Proposition 1, first consider a network in which all traders are arranged in a line (see Figure 1(b)). In this case, the resale value of intermediaries is ranked according to their distance to the final customer: the closer the intermediary is to the final customer, the higher is his resale value. Furthermore, the equilibrium payoffs of intermediary $i$ are simply $(1-p)$ times the resale value of $i$, and so the ranking of the equilibrium payoffs across intermediaries is the same as the ranking of their resale values. Finally,

\footnotetext{
${ }^{8}$ The proof of this result for general networks is more involved. To see why, note that a property of complete multipartite networks is that there are no paths connecting intermediaries in the same tier. Hence, whenever a seller in tier $x$ sells to an intermediary in tier $x+1$, the continuation payoff of all other intermediaries in tier $x+1$ is zero. In richer networks, the current seller may have neighbors $i$ and $j$, and $i$ and $j$ may be connected via another path, so that intermediary $j$ can still acquire the good after intermediary $i$ has purchased it. We refer to Manea (2013) for the general proof that deals with these subtleties.
} 
the equilibrium payoff of the initial seller is decreasing in the number of intermediaries, while the payoff of the final buyer is $v(1-p)$.

As the bargaining power shifts to upstream traders- i.e., $p$ increases - the payoff of the initial seller increases and the payoff of the final buyer decreases; the seller extracts all the surplus in the limit case where he has full bargaining power- i.e., $p \rightarrow 1$. In contrast, the payoff of each intermediary changes non-monotonically with $p$. It first increases with a shift of bargaining power to upstream traders, and then, eventually, decreases. Interestingly, for moderate values of $p$, it can be the case that an increase in $p$ increases the payoff of intermediaries who are close to the initial seller, but it decreases the rent for intermediaries that are close to the final customer.

Second, consider a competitive network (see Figure 1a). In this case, downstream intermediaries always compete for the object owned by the upstream intermediary. Competition at every level of the intermediation network implies that intermediaries have the same limiting resale value, regardless of their specific position. Furthermore, intermediaries obtain zero profit, and the seller and the buyer obtain the same payoff that they would obtain if they were to bargain directly-i.e., the seller obtains $p v$ and the final buyer $v(1-p)$.

When the network is competitive, horizontal mergers can be very profitable, as they can create substantial market power. For example, if all intermediaries in one tier decide to merge, the sum of their expected payoff would jump from 0 to $p v(1-p)$, whereas the seller's profits would decrease from $p v$ to $p^{2} v$, ceteris paribus. ${ }^{9}$

In describing Manea's (2013) framework we have made two important simplifications. First, we have assumed that there are no transaction costs. In the context of bilateral intermediation, if the seller $i$ has to pay a transaction cost $c$ when trading with $j$, a classical hold-up problem can emerge, giving rise to inefficiencies. ${ }^{10}$ Second, in complete multipartite networks, each intermediary in layer $x+1$ is identical from the view point of a seller in tier $x$. Hence, all paths are equally efficient. However, consider the network in Figure 2.1, and assume that the final buyer has a valuation of 1 and that each trader has a transaction cost $c$, which is assumed to be small, but positive. In order to maximize aggregate surplus, the object should flow from the initial seller to the final buyer via the shortest path, from $s$ to $b$ via intermediaries 3 and 4 . Instead, as Manea (2013) shows, for a high discount factor, in equilibrium, the seller trades with either intermediary 1 or intermediary 2 and the

\footnotetext{
${ }^{9}$ These conclusions are specific to the competitive network considered here; see Manea (2013) for a more general analysis of horizontal mergers. Manea (2013) also studies vertical mergers.

${ }^{10}$ For example, suppose that the seller $s$ is linked to intermediary $i$, who is linked to final buyer $b$; suppose, also, that the seller has a transaction cost of $c$ and the valuation of the buyer is $v>c$. Since the resale value of intermediary $i$ is $p v$, whenever $c>p v$, even if there are gains from trade, there is no trade in equilibrium. Wright and Wong (2014) study a similar model on a single chain of traders.
} 


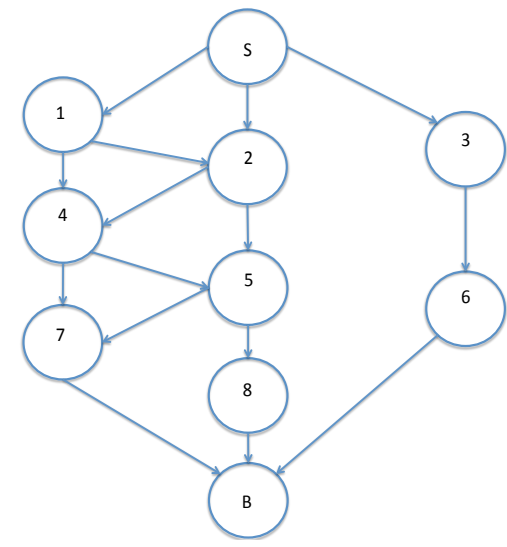

Figure 2: Trade does not minimize intermediation; Manea (2013).

object reaches the final buyer via at least three intermediaries. The key aspect here is that the competition in the network that the seller accesses via intermediaries 1 and 2 is more intense, relative to the competition in the network that the seller accesses via intermediary 3. This implies that intermediary 1's and intermediary 2's resale values are higher than intermediary 3's resale value, and it is, then, more profitable for the seller to bargaining with intermediaries 1 and 2 .

Siedlarek (2014) also studies a bargaining model in a directed network, but, building on Merlo and Wilson (1995), he focuses on a multilateral bargaining protocol. In each period, a path connecting the seller and the buyer is selected. Then, intermediaries in the path are ordered at random: the first intermediary makes a proposal, and, following the order, the other intermediaries sequentially accept or reject. The proposal is implemented if all intermediaries accept; otherwise, the game enters a new round of trade. Siedlarek (2014) characterizes stationary subgame perfect equilibria in a context in which different paths are allowed to generate different economic surplus. When the surplus of each path is homogeneous and agents become perfectly patient, the equilibrium characterization implies that only critical intermediaries obtain positive profit.

\subsection{Bilateral bargaining with asymmetric information}

The framework in Section 2.1 makes a neat distinction between buyers and intermediaries, and the consumption value of each trader is common knowledge. Condorelli and Galeotti 
(2012) propose a model of bilateral bargaining in networks with asymmetric information. ${ }^{11}$ All traders are potentially interested in consuming the object, and traders selling the good face incomplete information about the value of potential buyers. This framework is particularly appropriate for studying over-the-counter trading in financial markets, where, depending on private information, such as individual liquidity shocks, traders may buy for taking a certain market position or for reselling to other traders. Another interpretation is one of international trade, in which case the asymmetric information captures information frictions: the tastes of foreigns consumers may be very different from the tastes of domestic consumers. $^{12}$

More formally, the network is populated by a single initial seller $s$, and a number of traders, each with either a high consumption value $v_{H}$, or a low value $v_{L}$, with $0<v_{L}<v_{H}$. At the start of the game, the value of a trader is private information and the probability that trader $i$ has high value is $\pi_{i}$. The game develops in an infinite number of trading rounds, and traders discount the future at a common rate $1>\delta>0$. In each round, the owner of the object either consumes the object or makes a take-it-or-leave-it offer to a neighbor of his choosing, who, in turn, decides whether to accept or reject.

Asymmetric information considerably complicates the equilibrium analysis in this model. Condorelli and Galeotti (2012) provide a characterization of a specific class of stationary equilibria that they call "regular." 13 They show that all regular equilibria take the following form:

a. If the owner of the object has low valuation, he makes a sequence of take-it-or-leave-it offers to a subset of his neighbors, each at a price that makes a trader with high value indifferent between accepting and rejecting.

b. If all these offers are refused, the owner makes an offer at a price that equals the resale value of the trader receiving the offer. This offer is accepted with probability one by both the high- and low-value trader.

c. When a high-value trader acquires the object, he consumes it; when a low-value trader acquires the object, he resells it.

\footnotetext{
${ }^{11}$ Gofman (2011) builds a reduced-form model of trading in a network in which traders' valuations are private information and each intermediary receives an exogenous share of the gain from trade; the gain from trade is endogenously determined, based on the equilibrium decisions of agents to consume the object or resell it in the neighborhood.

${ }^{12}$ The importance of informational frictions in international trade is discussed in Chapter XX of the Handbook by Chaney.

${ }^{13}$ Roughly speaking, these are Weak Perfect Markov equilibria in which the strategy of a high-value buyer is constrained to be monotone in the price asked, for a certain region of the price support.
} 


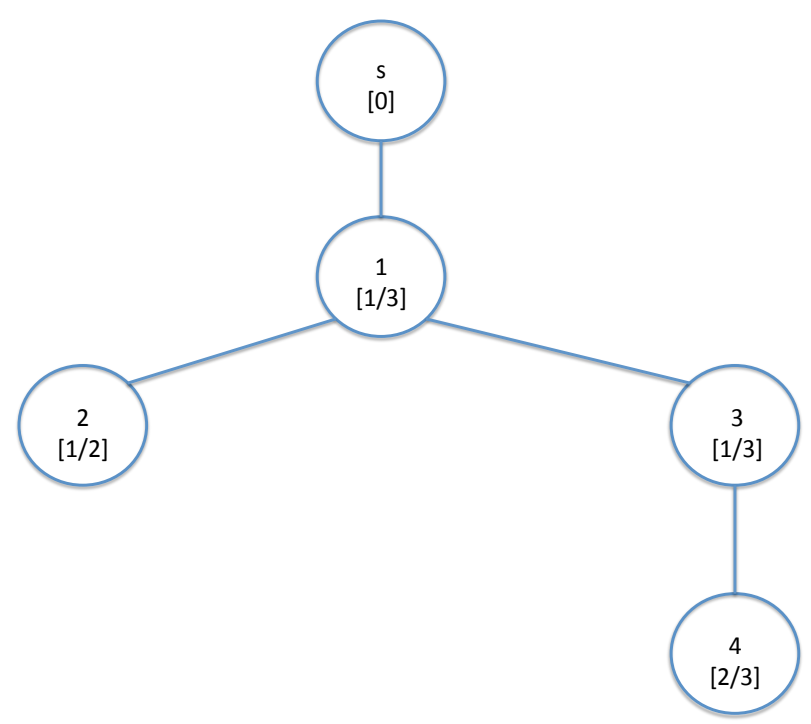

Figure 3: Examples of bargaining with asymmetric information.

d. The game continues in this fashion until a high-value trader acquires the object or a low-value trader decides to consume as the expected value of the object goes below $v_{L}$.

As an example, suppose that $v_{H}=1, v_{L}=0$, and consider the network in Figure 3; the number in the parentheses indicates the probability that the trader has high value. As agents become perfectly patient, the equilibrium path takes the following form: The initial seller offers the object to trader 1 at his resale value, which is $5 / 6$. Trader 2 accepts and consumes if he has high value; otherwise, he accepts and makes an offer of 1 to trader 2. Trader 2 accepts if he has a high value and otherwise rejects the offer. Upon rejection, trader 1 offers the object to trader 3 at his resale value, which is $2 / 3$. Trader 3 accepts the offer and consumes if he has a high value; otherwise, he makes an offer of 1 to trader 4 . If trader 4 has high value, he accepts and consumes; otherwise, trader 3 consumes.

Two main insights can be derived from this characterization. First, equilibrium asked prices are non-monotone in time: offers at the resale value decline over time, but the price asked between resale offers spikes upward. Offers at the resale value are declining over time because, as offers are rejected, traders learn that there are fewer and fewer potential customers in the network. The fact that prices spike from one resale offer to another reflects the attempt of intermediaries to exploit their local market power: they try to sell at a high price, and if they don't succeed, they lower the price and resell. ${ }^{14}$ In the example in Figure

\footnotetext{
${ }^{14}$ This is reminiscent of hot-potato trading strategies in OTC financial markets.
} 
3 , the sequence of prices asked is $\{5 / 6,1,2 / 3,1\}$.

Second, intermediaries who are essential to connect other intermediaries to the initial seller will make, in expectation, higher expected profits. Since resale offers are declining over time, receiving an offer later in the game allows an intermediary with a high value to obtain a higher profit margin. However, the later that an intermediary receives an offer, the higher is the ex-ante probability that the offer will not materialize, as earlier traders may consume the object. Condorelli and Galeotti (2012) show that the latter effect dominates the former. In the example above, only traders 1 and 3 obtain a positive profit, and their ex-ante expected payoff, condition on having a high value, is $1 / 6$ for trader 1 and $1 / 9$ for trader 3 .

Finally, for a given discount factor, it is easy to construct networks in which the equilibrium outcome is inefficient. But, as $\delta$ converges to one, in a stationary equilibrium, all offers are either at price $v_{H}$ or at the resale value of a trader, and the equilibrium outcome tends towards the ex-post efficient outcome. This result is in line with many dynamic models of bargaining with one-sided asymmetric information, such as Fudenberg, Levine and Tirole (1985) and Gul, Sonneschein and Wilson (1986).

\subsection{Multilateral bargaining through auctions}

We now move away from bilateral bargaining and consider trading protocols in which the seller more directly exploits competition across buyers. We build on Kotowski and Leister (2014), where in each round, the owner of the object sells it using a second-price auction. There is no asymmetric information about the valuation of final buyers, but each intermediary faces a random trading cost from purchasing an object. The trading cost can be either low, which we set equal to 0, or high, in which case it is higher than the consumption value to the buyer. Clearly, an intermediary can never recover a high cost of trade from buying and reselling, and, therefore, high-cost intermediaries will prefer not to trade. An intermediary's cost of trade is private information, and an intermediary has low cost with probability $p$.

We analyze the model within the class of complete multipartite networks. ${ }^{15}$ Trading occurs via a sequence of second-price, sealed-bid auctions: the initial seller $s$ runs an auction in which intermediaries in tier 1 bid; the winner, then, runs an auction in which intermediaries in tier $2 \mathrm{bid}$; and so on. We assume that the intermediary in the last tier $L$ who eventually owns the object sells it to the final customer at $v$. Intermediaries can bid any positive price

\footnotetext{
${ }^{15}$ The analysis of Kotowski and Leister (2014) applies to non-directed networks. They show that, even without the assumption that the graph is directed, there is an equilibrium in which goods flow from upstream firms to downstream firms.
} 
and can also abstain from the auction. Moreover, if only one intermediary makes a positive bid in one of the auctions, then he obtains the object at zero price. For simplicity, agents are assumed to be perfectly patient.

In this model of second-price auction with resale, there can be multiple equilibria. The focus is, then, on equilibria in which agents bid their expected resale value. Formally:

Proposition 2. Consider a complete multipartite network. There is an equilibrium where, in each auction in which the owner is an agent in tier $x \in\{0, \ldots, L-1\}$, high-cost intermediaries in tier $x+1$ do not participate in the auction, and low-cost intermediaries in tier $x+1$ bid their resale value, which is the asset's expected resale value conditional on all available information. Along the equilibrium path:

1. in the auction where the owner is an agent in tier $x \in\{0, \ldots, L-2\}$, the resale value of each low-cost intermediary in tier $x+1$ is

$$
r_{x+1}=\Pi_{y=x+2}^{L} \delta\left(n_{y}\right) v
$$

where $\delta\left(n_{y}\right)=1-(1-p)^{n_{y}}-n_{y} p(1-p)^{n_{y}-1}$ is the probability that at least two intermediaries in tier y have a low cost.

2. The ex-ante expected equilibrium payoff of an intermediary in tier $x$ is

$$
\pi_{x}=\Pi_{y=1}^{x-1}\left[1-(1-p)^{n_{y}}\right] \times p(1-p)^{n_{x}-1} \times r_{x}
$$

The proof of Proposition 2 follows from the main equilibrium characterization in Kotowski and Leister (2014). To understand the expression for the resale value, consider an intermediary that owns the object and that is located in tier $L-1$. All bidders in this auction have a resale value of $v$ by assumption and, therefore, will bid $v$ if they have a low cost. The expected profit of the intermediary owning the object is, then, the probability that at least two bidders have a low cost, $\delta\left(n_{L}\right)$, in which case he earns $v$. Proceeding backwards, we obtain the expression for the resale value.

The ex-ante payoff of an intermediary in tier $x$ is affected by: upstream concentration, horizontal concentration and downstream concentration. First, the higher the number of intermediaries in each upstream tier, the higher the chance that the object will reach tier $x-1$, which is a necessary condition for an intermediary in tier $x$ to make profit-i.e., first term in the expression. Second, the higher the number of intermediaries in each downstream tier, the higher downstream competition, and the higher the mark-up that the intermediary can obtain by buying and reselling-i.e., the last term in the expression. Finally, an intermediary 
in tier $x$ is better off when there are fewer intermediaries in his tier, as this improves his terms of trade in the auction- i.e., the middle term in the expression.

Node criticality is also important in this context. In fact, when an intermediary is critical, he will purchase the object at zero price. The market power that critical traders have relative to other intermediaries is, however, confounded by the uncertainty that the object flows along the different tiers and the uncertainty of the level of competition within each tier. By letting the probability that an intermediary has a low cost going to 1, we eliminate these uncertainties and obtain that only critical traders obtain a positive profit.

We now consider the effect of an horizontal merger. We make the natural assumption that the merged set of intermediaries operates at a low cost of trade if at least one of the intermediaries has a low cost.

Corollary 1. Suppose that in a complete multipartite network with tiers of size $\left\{n_{1}, \ldots, n_{L}\right\}$, a subset of intermediaries of size $\hat{n}_{x} \leq n_{x}$ in tier $x \in\{1, \ldots, L\}$ merge. By comparing the equilibrium characterized in Proposition 2 for the two networks, we obtain that:

1. The horizontal merger does not affect the aggregate expected surplus.

2. The horizontal merger increases the profit of each merged intermediary, it does not affect the profit of the other intermediaries in the same tier $x$ or in downstream tiers $\{x+1, \ldots, L\}$, but it decreases the expected payoff of the intermediaries in upstream tiers $\{1, \ldots, x-1\}$.

The aggregate surplus generated in equilibrium is $v$ whenever there is at least a trading path of intermediaries, each with a low cost of trade; otherwise, the realized surplus is zero. The assumption that the merged set of intermediaries operates at a low cost of trade if at least one of the intermediaries has a low cost implies that horizontal mergers do not alter the expected surplus. However, horizontal mergers change the way the surplus is distributed. A merger in tier $x$ reduces competition in that tier, and, therefore, each intermediary in tier $x+1$ anticipates that his resale value has decreased, and this extends to further upstream tiers. $^{16}$

Polanski and Cardona (2012) consider exchange through first-price auctions in a multilevel intermediation network. They study a multilevel symmetric tree, with a single seller

\footnotetext{
${ }^{16}$ Kotowski and Leister (2014) also discuss the effect of vertical mergers. They assume that in a vertical merger, the probability that the partnership operates at a low cost is the probability that there is at least one member intermediary in each tier with a low cost. This defines an implicit cost to vertical mergers, as it can decrease the probability that the partnership operates at a low cost. In some circumstances, this cost will be sufficiently large to make vertical mergers unprofitable.
} 
as the root of the tree, and buyers as terminal nodes. All buyers have independently distributed private values for the object held by the seller. Values are drawn from a uniform distribution. The intermediaries and the seller are interested only in their monetary payoff. In contrast to Kotowski and Leister (2014), their analysis is bottom-up: buyers with uncertain valuations submit bids to their intermediary. In turn, intermediaries submit their bids to the intermediary at the level above. Then, the processes propagates until it reaches the seller, who awards the object to the highest bidder among the intermediaries connected to him. Polanski and Cardona (2012) rank networks in terms of payoff for the seller offered by different configurations. Intuitively, for the same number of potential buyers, the seller prefers networks with fewer tiers of intermediation.

\subsection{Multilateral trading with posted bid-and-ask prices.}

We now turn to models in which intermediaries set a bid price to buy upstream and an ask price to sell downstream. Blume et al. (2007) introduce a bid and ask model in which each intermediary has no consumption value for the object and is connected to an arbitrary subset of buyers and sellers. Each seller has one unit of an homogeneous good, and each buyer demands one unit of the same good. The consumption value of buyers and sellers is arbitrary and commonly known.

The trading game in Blume et al. (2007) unfolds in two stages. First, each intermediary offers a bid price to each seller to whom he is connected, and an ask price to each buyer to whom he is connected. Second, sellers and buyers choose the best offer from the set of offers of traders connected to them. They may also choose not to sell or buy. A large penalty applies if intermediaries sell more units than they acquire, which guarantees that, in equilibrium, intermediaries will not default on their price commitment to buyers.

Blume et al. (2007) consider Nash equilibria of this game and show that all equilibria result in an efficient outcome-i.e., all possible beneficial trades are realized. They also show that a trader can make a positive profit if, and only if, the trader is essential. Here, a trader is essential if the social welfare decreases once the trader is removed from the network. When there are only one seller and one buyer, essentiality is equivalent to criticality.

By considering a setting in which there are only one buyer and one seller, it is possible to go beyond Blume et al. (2007) to consider complex networks, such as multipartite networks, in which all traders post bid-and-ask prices simultaneously. In this case, the object then flows from the initial seller to the highest bidder in tier 1 , say $i$, and from tier 1 to the highest bidder in tier 2, provided that such a bid meets intermediary $i$ 's ask, etc. The object stops flowing either when it is acquired by intermediary $i$ and $i$ 's ask is strictly higher than the 
highest bid of intermediaries in the downstream tier, or when it reaches the final customer b. Ties are resolved with randomization. This framework builds on Gale and Kariv (2009), and most of the results we present in this section are proved in Choi et al. (2013).

Proposition 3. Consider a complete multipartite network. There exists a Nash equilibrium that is efficient. In every efficient equilibrium:

\section{Only critical intermediaries can obtain positive profit.}

2. In the line network, all the surplus goes to intermediaries, whereas buyer and seller obtain zero profit. Indeed, any distribution of positive profits across intermediaries that sum up to $v$ can be sustained as an equilibrium outcome.

3. In a competitive network, all the surplus goes to the seller and the buyer, whereas intermediaries obtain zero profit.

The existence of efficient equilibria can be shown by construction. Consider the following bid/ask profile: each intermediary in tier $x=2 \ldots L$ bids $v$ and asks $v$; if there is only one intermediary in tier 1 then the intermediary bids 0 and asks $v$, if there is more than one intermediary in tier 1, then each intermediary bids $v$ and asks $v$. Under this profile, if an intermediary acquires the object, he will resell it to $v$. So, each intermediary is willing to bid up to $v$. Since each intermediary asks $v$, for every intermediary in tier $x>1$, it is a best reply to bid $v$. A monopoly intermediary in tier 1 will bid 0 , while if there are multiple intermediaries, competition will push bids up to $v$.

In the line network, the intermediary connected to the seller has full bargaining power and will extract all the resale value that his connection generates; similarly, the intermediary connected to the final customer also has full bargaining power, and so must also extract all the surplus from the buyer. Equilibrium does not pin down, however, how the total surplus $v$ is distributed across intermediaries. In fact, the problem faced by two consecutive intermediaries, $x$ and $x+1$, is akin to a Nash demand game where, though, the surplus that can be shared is endogenous and depends on the resale value of intermediary $x+1$. In a competitive multipartite network, competition across intermediaries at every level destroys intermediation rents, and the seller is the sole agent extracting all the surplus. As in the models we have analyzed previously, intermediaries who merge horizontally may increase their market power.

Gale and Kariv (2009) provide an experimental analysis of this model that focuses on competitive networks. They find that, after a period of learning, the bid-and-ask prices converge to the competitive equilibrium prices, and the outcome of trade becomes efficient. 
So, despite trade requiring possibly long chains of intermediaries, and the impossibility of recontracting, subjects are often able to coordinate on the efficient outcome. The experiment also points out that higher levels of competition across intermediaries, studied by increasing the number of intermediaries in different tiers, tend to speed up the learning and convergence towards efficient play. ${ }^{17}$

\subsection{Static trading protocols}

In all the papers we have surveyed so far, the market model contains an element of sequentiality, with sellers (or buyers) usually proposing the terms of trade and one or more buyers (or the seller) agreeing to trade according to their terms or refusing to trade. We now discuss three papers that, while maintaining that not everyone can trade with everyone else, exploit a more centralized/static equilibrium notion.

Nava (2009) studies the exchange in a network of a single homogeneous commodity. Traders' preferences are heterogeneous, and their utility is concave in the quantity of commodity consumed, while linear in money. The focus is on equilibrium in a simultaneous move game in which each trader decides how much quantity to sell to, or to buy from, other traders. As in a Cournot model, prices formed at every location reflect the local willingness to pay of a trader, given the demand and supply decisions in his local market. In equilibrium, there is intermediation, in that some traders buy some units of commodity in order to resell them to other traders. Prices strictly increase along any intermediation chain because a trader intermediates the commodity only if gains from trade are strictly positive. Efficiency is attained only in large economies and for specific network configurations. In particular, trade can be efficient only if intermediation is negligible in the economy. If intermediation is essential to clear markets, then a group of traders necessarily commands a rent that distorts trade.

Malamud and Rostek (2013) study a general model of asset trading, where connected traders participate in the same market. Traders simultaneously submit demand schedules, and pricing in each market is attained via a uniform-price double auction. Linear Nash equilibria are analyzed (following Kyle (1989) and Vives (2011)).

Babus and Kondor (2013) consider a setup closely related to that of Malamud and Rostek (2013). They focus on a less general connectivity structure, but they endow agents with idiosyncratic information on the asset's value and characterize the informational content of

\footnotetext{
${ }^{17}$ We refer to Chapter XX of the Handbook by Choi and Kariv for a detailed survey of experiments of networked markets. That chapter includes, among other related papers, an exhaustive discussion of Gale and Kariv (2009) and of Choi et al. (2013).
} 
prices. They show that information diffusion is effective, but not informationally efficient. They also show that dealers with more trading partners are ex post better informed, so they tend to trade and intermediate more, and earn more profit per transaction.

\section{Pricing in supply chains}

Section 2 focuses on pure intermediation: the role of intermediaries is to buy and resell assets, and assets flow from sellers to final customers. However, one can envisage an environment in which a chain of intermediaries is a final product, and the value of consumers for different paths may depend on the particular intermediaries within the path. For example, in supply chains, intermediaries within a tier provide substitute inputs, and intermediaries across tiers provide complementary inputs. Chains are, therefore, final products, each produced through the combination of a set of complementary inputs. Similarly, in the context of transportation networks, one can interpret a path as a journey and the intermediaries along the path as transport service providers.

There is renewed interest in supply chains in economics. The important role of production supply chains in trade has been documented empirically in different studies, such as Hummels et al. (2001). Antras and Chor (2013) study the optimal allocation of ownership rights along a supply chain. Oberfield (2013) develops a theory in which the network structure of production - who buys inputs from whom - is the endogenous outcome of individual choices. Acemoglu et al. (2012) focus on the role that production supply chains play in translating idiosyncratic shocks into volatility at the aggregate level.

The framework developed in this section, due to Choi et al. (2013) and Galeotti and Goyal (2014), abstracts from the role of idiosyncratic shocks in the value chain and from the endogenous formation of these networks. It complements the literature on supply chains by providing a systematic study of strategic pricing in competing chains. This work is also related to Acemoglu and Ozdgar (2007a, 2007b), who study the efficiency of oligopoly equilibria in congested markets, such as network flows in communication networks or traffic in transportation networks. ${ }^{18}$

Let $T$ be the set of directed paths connecting $s$ to $b$ in a complete multipartite network. Following Galeotti and Goyal (2014), we use a Dixit-Stiglitz framework to model paths as differentiated products that buyers may demand, see, also, Singh and Vives (1984). Let $p_{i}$ be the price for the service of intermediary $i$ and let $p=\left\{p_{1}, \ldots, p_{n}\right\}$ denote a price profile.

\footnotetext{
${ }^{18}$ Acemoglu and Ozdgar (2007a, 2007b) consider parallel paths networks. Another related paper is Hendricks, Piccione and Tan (1999), who study the problem of competing airlines each designing their transportation networks.
} 
The cost of a path $q \in T$ is the sum of prices charged by the intermediaries along that pathi.e., $c(q ; p)=\sum_{i \in q} p_{i}$. A representative consumer has quadratic utility over paths. That is, let $x_{q}$ be the consumption of a representative consumer for path $q \in T$, and let

$$
\hat{U}=\sum_{q \in T} x_{q}-\frac{\beta}{2} \sum_{q \in T} x_{q}^{2}-\frac{\gamma}{2} \sum_{q \in T} \sum_{q^{\prime} \in \mathcal{Q} \backslash\{q\}} x_{q} x_{q^{\prime}} .
$$

Then, the representative consumer maximizes $U=\hat{U}-\sum_{q \in T} c(q ; p) x_{q}$, where $\beta>0$ and $\gamma \in[0, \beta)$. Solving for optimum consumption leads to the following demand function for path $q$ :

$$
D(p, q) \equiv x_{q}=\frac{1}{\beta+\gamma(m-1)}\left[1-\frac{\beta+\gamma(m-2)}{(\beta-\gamma)} c(q ; p)+\frac{\gamma}{(\beta-\gamma)} \sum_{q^{\prime} \neq q} c\left(q^{\prime} ; p\right)\right],
$$

where $m$ is the total number of paths- i.e., $m \equiv|T|$. When $\gamma=0$ paths are independent goods, when $\gamma>0$ paths are substitute goods and, at the limit, when $\gamma \rightarrow \beta$, we have the case where paths are perfect substitutes. We report the Nash equilibrium of the following simultaneous move game: intermediaries set prices simultaneously and wish to maximize their individual profits, where intermediary $i$ 's profits is $\prod_{i}(p)=p_{i} \sum_{q \in T, i \in q} D(p, q)$.

The strategic relation between two intermediaries depends on their network location, together with the intensity of competition across paths. If intermediaries $i$ and $j$ share path $q$, then an increase in intermediary $i$ 's price decreases the demand of path $q$, and this creates incentives for intermediary $j$ to decrease his price. So, sharing paths create strategic substitutability between intermediaries' pricing strategy. In contrast, if intermediary $i$ is located in path $q$ and intermediary $j$ is located in path $q^{\prime}$, an increase in intermediary $i$ 's price decreases the competitiveness of path $q$ relative to path $q^{\prime}$, and intermediary $j$ can then raise his profits by increasing his price. Hence, belonging to different paths creates strategic complementarities between intermediaries' pricing strategy. As intermediaries may share some paths and not others, whether the prices of two intermediaries are strategic complements or strategic substitutes will depend on their specific network location.

The following result provides a characterization of equilibrium pricing and profits across intermediaries in complete multipartite networks. The betweenness centrality of intermediary $i$ is the ratio between the number of paths that $i$ belongs to and the total number of paths. In a complete multipartite network, the total number of trading paths is $m=\prod_{x=1}^{l} n_{x}$ and an intermediary in tier $y$ belongs to $\prod_{x=1, x \neq y}^{l} n_{x}$. Therefore, the betweenness centrality of an intermediary in tier $y$ is simply $1 / n_{y}$. 
Proposition 4. Consider a complete multipartite network. In equilibrium, the price and profit of an intermediary in tier $x$ is higher than the price and profit of an intermediary in tier $y$ if, and only if, the betweenness centrality of an intermediary in tier $x$ is higher than the betweenness centrality of an intermediary in tier $y$.

The result illustrates that betweenness centrality is an important determinant of market power for intermediaries. Betweenness centrality is formally related to the notion of node criticality. In fact, a node $i$ is critical if, and only if, has maximal betweenness centrality. When paths are imperfect substitute products, intermediaries enjoy rent even if they are not critical. As the next result illustrates, when paths become perfect substitutes, then only critical intermediaries charge a positive mark-up and obtain positive profits.

Proposition 5. At the limit, where paths become perfect substitutes-i.e., $\gamma \rightarrow \beta$ - the equilibrium price of each non-critical intermediary goes to 0, and the equilibrium price of each critical intermediary goes to

$$
p^{*}=\frac{\alpha}{k+1},
$$

where $k$ is the number of critical intermediaries. Hence, only critical intermediaries obtain positive profit.

Choi et al. (2013) provide a theoretical analysis of pricing in trading networks in which paths are perfect substitutes, and they provide experimental evidence of the model. In networks in which there are no critical intermediaries, their experimental results show that prices are generally very low, and the total cost of each chain is no larger than $20 \%$ of the consumers' willingness to pay. In contrast, when there are only critical intermediaries, intermediaries price similarly and, overall, the cost of the chain is above $90 \%$ of the consumers' willingness to pay. In the mixed cases, chains are priced similarly, and their cost is always above $60 \%$ of the consumer's valuation. Critical intermediaries price well above non-critical intermediaries and get most of intermediation's profits. Overall, the experimental results point to node criticality as an organizing principle for understanding pricing, efficiency and the division of surplus in networked markets.

We have discussed oligopolistic analysis of supply chains. Ovstrosky (2008) studies a complementary model of supply chains in networks. A set of firms is arranged in an acyclic directed network of trading possibilities. Each firm's profit depends on the contracts it can sign with upstream and downstream firms. An outcome is a set of contracts signed by firms; an outcome is stable if no firm would like to break any of its relationships or establish a new one with a willing partner. Ovstrosky (2008) develops conditions on preferences that assure the existence of stable outcomes. These conditions generalize the gross-substitute condition 
in Kelso and Crawford (1982). Insights of two-sided matching theory- e.g., Adachi (2000) and Hatfield and Milgrom (2005) - generalize besides existence: stable outcomes represent a lattice in the payoff space of firms at the top of the network-i.e., suppliers- and firms at the bottom-i.e., consumers. The generality of the model does not allow, though, to derive specific insights into the effect of the network structure on outcomes. ${ }^{19}$

\section{Discussion and open questions}

We conclude by discussing open questions in the literature. All the models discussed in Section 2 assume that there is only one seller with only one unit of the good. Extending the analysis to multiple units, heterogeneous supplies and heterogeneous demands can be a fruitful area for future research.

We have focused our attention on strategic intermediation in a given network and abstracted away from the equally important issue of trading network formation. Only a handful of papers are devoted to the study of network formation in an environment in which the benefits of linking are determined by an underlying game of trade. Goyal and Vega-Redondo (2007) characterize equilibrium networks under the assumption that only critical traders obtain positive payoff. Condorelli and Galeotti (2013) study network formation in a setting where, for every network, trade is efficient and there are no intermediation rents. In Section 3 , we have mentioned the work of Oberfield (2013), which develops a theory of endogenous input-output networks of production. Kotowski and Leister (2014), discussed in Section 2.3, characterize free-entry equilibrium networks. The formation of trading networks is a largely unexplored topic, and we believe it deserves further work.

The models we presented provide rich empirical predictions with respect to how the architecture of the network impacts efficiency, pricing, and distribution of economic surplus across traders. We discussed two recent experimental works on intermediation networks, i.e., Gale and Kariv (2009) and Choi et al. (2013). Both experiments exploit the simultaneous move nature of these two models, and, therefore, they abstract away from the possibility of re-contracting among intermediaries. We believe that adding a dynamic aspect to these experiments can be useful to evaluate the insights that the theory provides on intermediation in networks.

Finally, it is well known that bargaining games are sensitive to the specification of the extensive form-i.e., the trading protocol. This is even more so when there are more than two

\footnotetext{
${ }^{19}$ Hatfield et al. (2014) generalize Ovstrosky's (2008) to arbitrary networks- i.e., allowing for inter-dealer trading in addition to upstream and downstream trading.
} 
bargaining parties as in intermediation networks. Understanding optimal trading protocols from the perspective of the various actors involved in the network is an open research agenda.

\section{References}

[1] Acemoglu, D, V.M. Carvalho, A. Ozdaglar and A.Tahbaz-Salehi (2012), The Network Origins of Aggregate Fluctuations, Econometrica, 80 (5), 1977-2016

[2] Acemoglu, D. and A. Ozdaglar (2007a), Competition in Parallel-Serial Networks, IEEE Journal on selected areas in communications.

[3] Acemoglu, D. and A. Ozdaglar (2007b), Competition and Efficiency in Congested Markets, Mathematics of Operations Research, 32, 1, 1-31.

[4] Adachi, H. (2000), On a Characterization of Stable Matchings, Economics Letters, 68(1): 43-49.

[5] Antras, P., and D. Chor (2013), Organizing the Global Value Chain, Econometrica, 81, $6,2127-2204$.

[6] Babus, A., and P. Kondor (2013), Trading and Information Diffusion in OTC Markets. Mimeo, CEU. Budapest.

[7] Blume, L., D. Easley, J. Kleinberg and E. Tardos (2007), Trading Networks with PriceSetting Agents, in Proceedings of the 8th ACM conference on Electronic commerce EC 2007 New York, NY, USA.

[8] Choi, S., A. Galeotti and S. Goyal (2013), Trading in networks: theory and experiment. Mimeo.

[9] Condorelli, D. and A. Galeotti (2012), Endogenous Trading Networks. Mimeo.

[10] Condorelli, D. and A. Galeotti (2012), Bilateral Trading in Networks. Mimeo.

[11] M. Corominas-Bosh (2004), Bargaining in a network of buyers and sellers, Journal of Economic Theory, 115, 35Đ77.

[12] Duffie, D., N. Garleanu, and L.H. Pedersen (2005), Over-the-Counter Markets, Econometrica, 73, 1815- 1847.

[13] Fafchamps, M. and B. Minten (1999), Relationships and Traders in Madagascar, The Journal of Development Studies, 35, 6, 1-35. 
[14] Fudenberg, D.; Levine, D. K. and Tirole, J. Roth, A. (Ed.) Bargaining with Incomplete Information Infinite-Horizon Models of Bargaining with One-Sided Incomplete Information Game Theoretic Models of Bargaining, Cambridge University Press, 1985, 73-98

[15] Gale, D. and S. Kariv (2009), Trading in Networks: A Normal Form Game Experiment, American Economic Journal: Microeconomics, 1, 2, 114-32.

[16] Galeotti, A and S. Goyal (2014), Competing chains. Mimeo.

[17] Gofman, M (2011), A Network-Based Analysis of Over-the-Counter Markets. Mimeo Wisconsin-Madison.

[18] Gul, Faruk, Hugo Sonnenschein, and Robert Wilson (1986), Foundations of dynamic monopoly and the coase conjecture, Journal of Economic Theory, 39 (1), $155 Đ 190$.

[19] Goyal, S. and F. Vega-Redondo (2007), Structural Holes in Social Networks, Journal of Economic Theory, 137, 460-492.

[20] Hatfield, J. W., and Milgrom, P.R. (2005), Matching with Contracts, American Economic Review, 95(4): 913-35.

[21] Hatfield, J.H., S.D. Kominers, A. Nichifor, M. Ostrovsky, and A. Westkamp (2013), Stability and Competitive Equilibrium in Trading Networks, Journal of Political Economy, 121, 966-1005.

[22] Hummels, D., J. Ishii and K.-M. Yi (2001), The Nature and Growth of Vertical Specialization in World Trade, Journal of International Economics, 54, 1, 75-96.

[23] Kirman, A. (1997), The economy as an evolving network, Journal of Evolutionary Economics, 7(4), 339-353

[24] Kyle, A. S. (1989), Informed Speculation and Imperfect Competition, Review of Economic Studies, 56, 517-556.

[25] Kotowski, M. H. and C. M. Leister (2014), Trading Networks and Equilibrium Intermediation. Mimeo.

[26] Kranton, R. and D. Minehart (2001), A Theory of Buyer-Seller Networks, American Economic Review, 91, 485-508.

[27] Li, D. and N. Schurhoff (2012), Dealer Networks. Mimeo, University of Lausanne. 
[28] Malamud, S. and M. Rostek (2013), Decentralized Exchange, Mimeo, Wisconsin Madison.

[29] Manea, M. (2013), Intermediation in Networks. Mimeo.

[30] Merlo, A. and C. Wilson (1995), A Stochastic Model of Sequential Bargaining with Complete Information, Econometrica 63(2), 371-399.

[31] Nava, F. (2010), Flow Competition in Networked Markets. Mimeo.

[32] Oberfield E. (2013), Business Networks, Production Chains, and Productivity: A Theory of Input-Output Architecture. Mimeo.

[33] Ostrovsky, M. (2008), Stability in supply chain networks, American Economic Review, 98:3 897-923.

[34] Polanski A. and Cardona D. (2012), Multilevel Mediation in Symmetric Trees, Review of Network Economics, 11(3), 1-23.

[35] , Rauch J. (1999), Networks versus Markets in International Trade, Journal of International Economics, 48, 7-35.

[36] Rubinstein, A. (1982), Perfect Equilibrium in a Bargaining Model, Econometrica, 50 (1), 97Đ109.

[37] Rubinstein, A. and A.Wolinsky, (1987) Middlemen, The Quarterly Journal of Economics, 102 (3), 581-93.

[38] Siedlarek, J.-P. (2014), Exchange with Intermediation in Network. Mimeo.

[39] Spulber, D. (1999), Market Microstructure: Intermediaries and the Theory of the Firm.

[40] Tesfatsion, L. (1997), A trade network game with endogenous partner selection, in Computational approaches to economic problems, Springer.

[41] Vives, X. (2011), Strategic Supply Function Competition with Private Information, Econometrica, 79, 6, 1919-66.

[42] Wright R. and Wong Y. (2014), Buyers, sellers, and middlemen: Variations on searchtheoretic themes, International Economic Review, 55(2), 375Đ397. 